Itinéraires Itinéraires

Littérature, textes, cultures

\title{
Mohamed Leftah : le corps dans l'ordre poétique
}

\section{Ziad Elmarsafy}

\section{OpenEdition}

\section{Journals}

Édition électronique

URL : http://journals.openedition.org/itineraires/1513

DOI : 10.4000/itineraires.1513

ISSN : 2427-920X

Éditeur

Pléiade

Édition imprimée

Date de publication : 1 novembre 2011

Pagination : 75-87

ISBN : 978-2-296-55720-8

ISSN : 2100-1340

Référence électronique

Ziad Elmarsafy, « Mohamed Leftah : le corps dans l'ordre poétique », Itinéraires [En ligne],

2011-3 | 2011, mis en ligne le 01 novembre 2011, consulté le 02 mai 2019. URL : http://

journals.openedition.org/itineraires/1513 ; DOI : 10.4000/itineraires.1513

Itinéraires est mis à disposition selon les termes de la licence Creative Commons Attribution - Pas d'Utilisation Commerciale - Pas de Modification 4.0 International. 


\title{
Mohamed Leftah : le corps dans l'ordre poétique ${ }^{1}$
}

\begin{abstract}
The article proposes a reading of the body in the work of Moroccan novelist Mohamed Leftah (1946-2008). The reading is built around three axes: the body as a locus of signification, the corporeal imaginary as encoded in Leftah's naturalism, and finally the status of the body within the contemporary political framework. Through Leftah's use of eros, violence and body art, and relying on the work of Peter Brooks, Gilles Deleuze, Félix Guattari and Alfred Gell, the article makes a case for the sublation of the corporeal into the poetic as being an urgent political and ethical task.
\end{abstract}

Keywords : Leftah, Morocco, tattoo, body in literature, erotic literature

Mots clés : Leftah, Maroc, tatouage, le corps dans la littérature, littérature érotique

L'ouverture de Demoiselles de Numidie, le premier roman de Mohamed Leftah qui raconte la vie d'un bordel casablancais, contient le passage suivant :

De cette violence, de cette nuit, de quelques mots matriciels et mystérieux : fleurs, filles, chancre, pollen, stigmates; de certains noms enchantés : Yasmine, Zoumourrod, arriverai-je à tirer un chant? À dégager un ordre? Poétique.

Le seul ordre acceptable ${ }^{2}$.

La tâche de l'écriture est d'imposer cet ordre au monde à travers un traitement littéraire du corps, ce dernier étant l'aspect le plus notable de l'œuvre leftahienne. Notre lecture propose un examen des corps qui

1. Je tiens à remercier Marc Kober et Véronique Bonnet pour l'organisation du colloque où cet article fut prononcé dans une première version. Je tiens à remercier aussi Anouar Ouyachchi qui m'a persuadé de lire Mohamed Leftah, et Jane Elliott pour des conseils d'une très grande valeur.

2. Mohamed Leftah, Demoiselles de Numidie [1992], Paris, La Différence, 2006, p. 11. 
remplissent les textes de Leftah à travers trois axes : le corps comme lieu de signification, l'imaginaire du corps entre naturalisme et esthétisme, et le corps dans le cadre politique.

\section{Le corps comme lieu de signification}

Le travail de Peter Brooks nous sert de point de départ. Dans Body Works, Brooks propose le corps comme locus de signification littéraire. Le corps est dans le roman et fait partie $d u$ roman : il est là pour être lu, compris, interprété. La vérité est inscrite sur et dans le corps, et ne se révèle qu'en le faisant parler :

Modern narratives appear to produce a semioticization of the body which is matched by a somatisation of story : a claim that the body must be a source and locus of meanings, and that stories cannot be told without making the body a prime vehicle of narrative significations ${ }^{3}$.

[La narration moderne produit une sémiotisation du corps qui est accompagnée d'une somatisation (ou une incorporation) du récit : elle prétend que le corps doit être la source et le lieu de la signification, et que les histoires n'ont lieu qu'en faisant du corps le moteur primaire de la signification narrative.]

Cet argument pourrait servir de principe structurel chez Leftah, pour qui la multiplication et la destruction des corps deviennent ce moteur de la narration. L'ubiquité du corps dans le texte de Leftah sert à rappeler au lecteur la matérialité commune à l'être et à l'écriture. Ainsi prostituées et proxénètes, gardes et prisonniers, tortionnaires et victimes envahissent son œuvre, tantôt déclarant qu'ils ne sont que des êtres de papier ${ }^{4}$, tantôt poussant la violence à la limite du supportable, pour constituer le corpus littéraire leftahien.

Cette constitution est comme cimentée par une autre constante de l'écriture de Leftah : l'intertextualité. On trouve partout des allusions, des références, des dialogues avec d'autres écrivains et artistes : Stendhal, dont Le Rouge et le Noir devient un leitmotiv dans Hawa, Lermontov, qui prête un titre à Leftah (Un martyr de notre temps), Dostoïevski, dont Les Possédés inspirent la cellule terroriste du Jour de Vénus, ainsi que Baudelaire, Verlaine, Rimbaud. On trouve également les poètes pré-islamiques et islamiques (Imru` Al-Qays, Abū Firās Al-Ḥamadānī) et divas arabes du $\mathrm{Xx}^{\mathrm{e}}$ siècle, Fairouz et Umm Kalthoum. Tant de noms pour dénombrer les éléments dont l'idiome de Leftah est fabriqué. Selon Khalid Zekri, cette

3. Peter Brooks, Body Work : Objects of Desire in Modern Narrative, Cambridge, MA, Harvard University Press, 1993, p. xii.

4. Le meilleur exemple ici serait peut-être la réflexion pirandellienne qui clôt Un martyr de notre temps, "L'Auteur en quête de personnage ", Un martyr de notre temps, Paris, La Différence, 2007, p. 77-91. 
pratique est liée à une stratégie qui relève autant de la génétique du roman que de l'histoire littéraire :

À travers le dialogue avec d'autres monuments littéraires, le texte prend naissance pour mieux instituer sa littérarité à travers la mémoire littéraire de l'autre. Plus il est dialogique, plus il s'ouvre a une pensée de l'altérité, et partant, renforce ses stratégies de séduction qui mettent l'accent sur son besoin de s'inscrire dans le discours d'autrui tout en le transformant ${ }^{5}$.

Ce qui veut dire que pour Mohamed Leftah, le texte lui-même doit faire corps avec d'autres textes littéraires. Chaque ligne, chaque mot, doit en attirer d'autres, pas pour les imiter, mais pour s'inscrire avec eux dans le même corpus, se les approprier et les transformer, pour mettre sur le même pied Fairouz et Baudelaire, Umm Kalthoum et Stendhal. L'allusion déjoue les différences entre textes orientaux et occidentaux; tout est posé sur le même plan. Ce n'est pas par hasard que Leftah met en exergue d'une nouvelle cette phrase de Levinas : " L'écriture rechercherait comme d'emblée la poétique qui est probablement le rassemblement des choses dans un monde où tout prend sens ${ }^{6}$. " Ce rassemblement revendique l'immanence de l'écrit.

Regardé de plus près, le texte leftahien est un texte où le corps parle pour exprimer sa vérité, aussi difficile ou inacceptable qu'elle puisse être. Considérons Fatima, une barmaid casablancaise qui est « ramassée » par Khabir, un commissaire des Renseignements généraux. Khabir amène Fatima dans sa villa du quartier de l'Anfa. Il la viole, et l'acte est précédé d'une déclaration symptomatique; il lui dit : « je le ferai parler ton lssan, poufiasse ${ }^{7} \gg$. Or lssan, en arabe, signifie langue, mais comme nous l'explique Mohamed Leftah, " c'est de ce terme qu'on appelle en arabe dialectal le clitoris ${ }^{8} »$. Faire parler le lssan de Fatima c'est donc l'obliger à assumer, à exprimer un désir qui n'est pas le sien. Ce qui arrive : Fatima « haletante, à bout de souffle cria son désir de sexe », le commissaire la pénètre, et Fatima, " les larmes aux yeux, cria son orgasme et alors que celui-ci l'emportait encore dans son déferlement, elle ferma les yeux et jura qu'elle vengerait ce viol. Ces larmes ${ }^{9} \gg$.

Le dernier mot de ce passage fait allusion à la chanson que Fatima chante sans cesse dans son bar. En l'occurrence c'est l'ode célèbre du poète syrien du $\mathrm{X}^{\mathrm{e}}$ siècle, Abū Firās, que Leftah traduit dans le texte de la nouvelle :

5. Khalid Zekri, Fictions du Réel. Modernité romanesque et écriture du réel au Maroc, Paris, L'Harmattan, 2006, p. 133.

6. Mohamed Leftah, Un martyr de notre temps, op. cit., p. 77.

7. Mohamed Leftah, Récits du monde flottant, Paris, La Différence, 2010, p. 56.

8. Ibid., p. 56.

9. Ibid. 
Je te vois, stoïque et inaccessible aux larmes

La loi de l'amour n'aurait-elle donc aucune emprise sur toi?

Oui je souffre et aspire au bien-aimé, mais les êtres

À ma semblance ne divulguent point leur secret ${ }^{10}$.

Le secret en question est l'amour que Fatima garde pour son fiancé, mort dans un accident d'avion. Ce qui frappe le lecteur, pourtant, est le fait que le commissaire, ce parangon du sadisme, clame ces vers lorsqu'il voit Fatima dans le bar, et d'ajouter, une fois qu'ils sont au palais : " personne n'est inaccessible aux gémissements et aux pleurs. Ni ton poète antédiluvien d'Alep, ni ce vieux nostalgique d'un temps révolu, ni toi, grande poufiasse ${ }^{11} »$, manifestant simultanément sa culture et sa vulgarité. De fait, il s'agit, pour le commissaire, de prouver l'hypothèse qu'il prononce avec le corollaire que la production des pleurs et des gémissements peut être stimulée quels que soient les moyens. Ce qui est démontré. La démonstration entraîne la trahison du corps et des désirs de Fatima, qui constate avec horreur qu'elle répond sexuellement à la stimulation d'un tortionnaire et d'un violeur. Refusant de vivre avec cette réalité d'un corps insoutenable, et dont la partie la plus intime finit par lui échapper, elle égorge le commissaire, ce meurtre étant le seul moyen de rester inaccessible aux larmes.

Si le corps de Fatima parle malgré lui, le corps de Zapata, l'un des « macs » héroïques, les anges bagarreurs de Leftah, et un personnage central dans Hawa et Demoiselles de Numidie, parle à dessein, à travers des images. Le corps de Zapata est orné de deux grands tatouages, un tigre et une ancre. Quand Ingvar, un marin norvégien amoureux de Zapata, vient lui rendre visite, il subit les effets de ces images. Regardant Zapata, il s'arrête sur place, terrorisé :

C'est que de l'échancrure de la chemise noire de Zapata, le tigre tatoué sur le torse dans des tons bleu sombre, sa gueule grande ouverte sur des crocs étincelants, lui sembla prendre son élan et s'apprêter à sauter sur lui pour le déchiqueter. De même, la verge d'acier de l'ancre tatouée dans les mêmes tons sur le buste, lui avait paru s'animer et quitter le support auquel elle était fixée pour s'abattre sur lui et le fendre ${ }^{12}$.

De métaphoriques, les tatouages deviennent des métonymies qui annoncent le destin d'Ingvar lors de sa rencontre érotique avec Zapata, rencontre pendant laquelle il est « déchiqueté » et « fendu ». L'importance de cette métonymie est soulignée par Leftah dans sa description du corps à corps de l'acte sexuel : Ingvar se retrouve tout nu, étendu par terre, avec « un torse tatoué plaqué sur son $\operatorname{dos}^{13} »-$ ce n'est pas simplement

10. Ibid., p. 51.

11. Ibid., p. 54.

12. Mohamed Leftah, Hawa, ou le chant du quartier Boussbir, Paris, La Différence, 2010, p. 57.

13. Ibid., p. 57. 
le corps de Zapata qui importe ici, mais les tatouages à travers lesquels ce corps s'exprime.

De même, lorsqu'il fait l'amour avec le capitaine du navire, celui-ci déchiffre et interprète le corps de Zapata, qui devient comme le reflet de l'image qu'il porte :

Il [le capitaine] s'avança, sa main droite se posa, tremblante, sur le torse bombé, l'index de cette main, délicatement, commença à suivre lentement le contour de l'ancre en acier forgé, d'abord le jas, puis la verge sur laquelle cette pièce s'articulait perpendiculairement, qu'il parcourut d'une extrémité a l'autre, l'organeau aux deux bras armés de pattes, de cette verge l'index descendit tout aussi lentement [...] c'était la première fois que le capitaine exécutait manœuvres d'ancre aussi inédites, aussi délicates, mais qu'il réussissait miraculeusement et à la perfection, la verge de Zapata, qu'on eut dite elle aussi en acier forgé, se métamorphosant en pistil vivant qui, comme en une corolle de fleur, s'épanouissait maintenant, palpitant, dans la bouche du capitaine ${ }^{14}$.

Le corps est lu d'abord, et puis approprié, ingéré.

Cette séduction par le tatouage nous fournit un point de contact important entre les romans de Leftah et les écrits de Deleuze et Guattari. Ingvar voit le tatouage, le touche et se laisse toucher par lui. L'intimité permet la vision de près qui se rapproche du toucher : l'œil touche autant qu'il voit, l'optique devient haptique, le visuel devient tactile ${ }^{15}$. L'objet de cette vision rapprochée, l'espace lisse ${ }^{16}$, correspond au tatouage sur le corps de Zapata. Dans un article récent, Andrea Flores Khalil compare l'usage que fait Abdelkébir Khatibi du tatouage à l'espace lisse deleuzien, montrant comment le tatouage libère l'écriture des espaces striés par des investissements idéologiques contradictoires ${ }^{17}$. Chez Leftah, par contre, l'espace lisse qui correspond au tatouage libère les intensités du corps. Le corps des deux amants, Ingvar et Spartacus, est projeté vers son destin orgasmique par la médiation du tatouage.

\section{Ibid., p. 59-60.}

15. Gilles Deleuze et Félix Guattari, Mille plateaux. Capitalisme et schizophrénie 2, Paris, Minuit, 1980, p. 598, p. 614-616. Nous suivons l'usage deleuzien du mot « haptique » pour désigner tout ce qui a trait au toucher. Il convient de signaler ici l'analyse que fait Derrida de ce texte pour démontrer l'impossibilité du contact " immédiat » avec et à travers le toucher ainsi que le commentaire lucide de J. Hillis Miller sur cette thèse. Jacques Derrida, Le Toucher, Jean-Luc Nancy, Paris, Galilée, 2000, p. 141-45; J. Hillis Miller, « Touching Derrida Touching Nancy : The Main Traits of Derrida's Hand », Derrida Today, vol. 1, nº 2 , nov. 2008, p. 155. Nous irions plus loin : chez Leftah c'est l'art qui doit servir d'intermédiaire entre les corps.

16. Nous suivons la description de Deleuze et Guattari : «C'est le Lisse qui nous paraît à la fois l'objet d'une vision rapprochée par excellence et l'élément d'un espace haptique (qui peut être visuel, auditif autant que tactile). », op. cit., p. 615.

17. Andrea Flores Khalil, «A Writing in Points : Autobiography and the Poetics of the Tattoo », Journal of North African Studies, vol. 8, n² 2, 2003, p. 22, p. 30-32. 
Cette projection du corps passe par un autre élément deleuzien. Car le tigre de Zapata n'est pas qu'un tatouage : il s'agit plutôt d'un devenir ${ }^{18}$. De ce point de vue le tatouage ne serait plus une simple « imitation » mais un devenir-tigre qui a lieu lors de la rencontre érotique entre Ingvar et Zapata ${ }^{19}$. À travers ce devenir il y a une multiplication, voire une reproduction de Zapata-qui-devient-tigre. La pratique du tatouage, comme celle de toute inscription du corps, a depuis longtemps servi de moyen de la reproduction de l'identité ${ }^{20}$. Leftah met ce lien en valeur dès le début de sa carrière, quand il parle des cicatrices qui définissent la profession et le rang des « femmescicatrices », les prostituées de Casablanca ${ }^{21}$. Tant chez les prostituées que chez Zapata, l'art - sous forme de tatouage ou de cicatrice - dépasse sa fonction décorative pour devenir un moyen de « distribuer » la personne, d'agrandir sa présence, d'augmenter ses pouvoirs ${ }^{22}$. Cette augmentation rappelle la théorie épicurienne des simulacres volants : les corps émettent des images qui émanent de leur surface pour voyager dans l'univers ${ }^{23}$. De même, le torse tatoué qui est plaqué sur celui d'Ingvar produit une émanation semblable pour marquer l'amant norvégien du simulacre de Spartacus, dont le nom même résulte d'une autre émanation, d'un autre simulacre volant en l'occurrence le film Viva Zapata ${ }^{24}$. La multiplication et la distribution du corps, comme devenir et simulacre, est inscrite dans le nom du personnage.

Cette multiplication du proxénète continue le processus de deveniranimal. Deleuze nous rappelle que « tout animal est d'abord une bande, une meute $^{25} \gg$. Zapata n'est pas le seul personnage de Leftah à subir ce devenir. Dans « La Petite chamelle du bon dieu », Fakhita, 1'épouse d'un boucher et propriétaire d'une ferme, fait un rêve où elle devient une chamelle que

18. Gilles Deleuze et Claire Parnet, Dialogues, Paris, Flammarion, 1996, p. 8-10.

19. Deleuze et Guattari (op. cit., p. 374) vont jusqu'à refuser la mimésis : " Aucun art n'est imitatif, ne peut être imitatif ou figuratif : supposons qu'un peintre "représente" un oiseau; en fait, c'est un devenir-oiseau qui ne peut se faire que dans la mesure où l'oiseau est lui-même en train de devenir autre chose, pure ligne et pure couleur. »

20. Alfred Gell, Wrapping in Images : Tattooing in Polynesia, Oxford, Clarendon Press, 1993, p. 8; Nikki Sullivan, Tattooed Bodies : Subjectivity, Textuality, Ethics and Pleasure, Westport, CT, Praeger, 2001, p. 15-17, et pour une synthèse anthropologique plus générale, Enid Schildkrout, «Inscribing the Body », Annual Review of Anthropology, n 33, 2004, p. 319-344.

21. Mohamed Leftah, Demoiselles..., op. cit., p. 10-11.

22. Nous faisons allusion à la théorie de la « distributed person » utilisée par Alfred Gell pour expliquer la fonction instrumentale de l'art (selon laquelle l'œuvre d'art serait un agent, un acteur social) dans ses rapports avec la sorcellerie. Art and Agency : An Anthropological Theory, Oxford, Clarendon Press, 1998, p. 16-23, p. 96-154.

23. Cf. De rerum natura, IV, 30-127. Lucrèce utilise le mot « simulacrum » pour traduire l'eidolon (l'idole) d'Épicure. Le rapport d'Ingvar au corps tatoué de Spartacus, ne serait-il pas une forme d'idolâtrie?

24. Mohamed Leftah, Hawa ..., op. cit., p. 15.

25. Gilles Deleuze et Félix Guattari, op. cit., p. 293. 
son époux a égorgée ${ }^{26}$. Le surlendemain elle commence à s'appeler Jmiyla (petite chamelle) et répète les gestes de cet acte : elle se caresse habituellement le cou, la tête légèrement tournée de côté, comme si elle se présentait pour être égorgée elle-même. Elle finit ses jours dans « une tente dressée prés de l'enclos des camélidés », ayant intégré la meute ${ }^{27}$. Le narrateur d'une autre nouvelle, « Des chiens et des hommes », avoue avoir fait partie d'une bande de voyous impliquée dans un viol collectif. Le tout est raconté sous forme d'une aventure d'une meute de chiens (Leftah utilise le mot « meute » onze fois dans une nouvelle de cinq pages). Ayant fait le tour de plusieurs incidents de barbarie canine, le narrateur se demande :

Mais fallait-il en vouloir aux chiens s'ils redevenaient sauvages, sortaient leurs crocs pour terroriser l'homme ou le déchiqueter? Ce dernier n'était-il pas à l'origine de leur ensauvagement? Lequel des deux était plus chien que l'autre ${ }^{28}$ ?

Plusieurs années après, les chiens de la meute passent par d'autres devenirs : le premier, l'ancien mâle dominant, devient un alcoolique, le deuxième un artiste raté et amer, le troisième un douanier, le quatrième un vétérinaire et le cinquième le narrateur de la nouvelle. Tous - hommes et chiens - sont pris dans ces blocs de devenir qui inscrivent le corps dans une multiplicité, qu'elle soit une meute de chiens ou la société casablancaise.

Le devenir du corps sert de devise dans l'œuvre de Leftah. Dans une nouvelle qui raconte la mort d'Héraclite, «Le Philosophe dévoré par les chiens ", le vieux philosophe, atteint d'hydropisie, se couvre de bouse pour atténuer la douleur. Il est attaqué et tué par une meute de chiens. Abandonné par les dieux et agressé par les êtres familiers, l'homme n'est qu'une « infime et éphémère gouttelette dans le flot du devenir ${ }^{29}$ ». Leftah établit un parallèle entre ce destin et le début de la nouvelle, épisode au cours duquel le philosophe regarde un adolescent se baigner dans la mer - « Le flot, le flux du devenir! 》- avant de se retrouver « écrasé » par « ce devenir universel ${ }^{30} »$. Tout corps est voué à se dissoudre dans le devenir.

\section{Naturalisme/esthétisme}

Zapata possède un devenir-tigre mais passe par d'autres devenirs. En tant qu'homme animal, il a un rapport privilégié avec l'inceste ${ }^{31}$. Chaque

26. Dans «L'Auteur en quête de personnage », Leftah explique qu'il s'agit d'une allusion au récit coranique du prophète Sālih et le peuple de Thamūd. Leftah, Un martyr..., op. cit., p. 86-87. Cf. Le Coran, sourate XCI, v. 11-14.

27. Mohamed Leftah, Un Martyr..., op. cit., p. 46.

28. Ibid., p. 61.

29. Mohamed Leftah, Récits..., op. cit., p. 10.

30. Ibid., p. 10.

31. Deleuze et Guattari, op. cit., p. 303 n. 
aspect de son histoire est accompagné d'un chant récité par Hawa, sa sœur, avec qui il entretient un rapport incestueux. Le corps de l'ange bagarreur est doublé par l'art du chant. De plus, le style de ces chants comporte une multiplication, effectuée à travers l'allitération et l'anaphore, comme si le style du chant devait reproduire le rapport entre Zapata et sa sœur. Avant de devenir tigre, Zapata est pris dans un autre devenir à travers la comparaison traditionnelle entre le mâle hyper-viril et l'étalon (al-fahl, lafhal). À ce propos Leftah ne manque pas de nous rappeler la célèbre description du cheval tirée de l'ode suspendue d'Imru'Al-Qays : «Attaquant battant en retraite, avançant reculant dans le même / mouvement tel un bloc chu roulé par une cataracte ${ }^{32}$. » Lors d'une rixe entre Zapata et Spartacus (un autre proxénète), Hawa chante la force de son frère-cheval : « Lefhal va hennir de hennissement / Lafhal va avancer d'avancement [...] Les lèvres de l'étalon ont écumé d'écume / d'écume coléreuse elles ont écumé ${ }^{33}$. » Le dernier chant de Hawa, produit à l'occasion de la mort de Zapata, poursuit les devenirs de celui-ci via l'identification à la poétesse Al-Kahnsā' et à son frère Saqr (le faucon) : «Je ne te pleurerai pas, frère aimé, comme Al-Khanssa pleura son frère / au nom de faucon, Saqr! Je ne te pleurerai pas, toi qui fus mon faucon et mon tigre ${ }^{34}$. » Le dédoublement entre Zapata et Hawa, entre l'art et la vie, entre le corps physique et le corps esthétique représenté par les tatouages de Zapata et les chants de Hawa, pousse le roman vers sa conclusion.

À ce dédoublement vient s'ajouter un autre rapport spéculaire, où les événements du roman reflètent le texte même d'une allusion. Hawa, comme d'habitude, annonce par son chant les événements qui auront lieu pendant les rencontres entre Zapata, Ingvar et le capitaine anonyme :

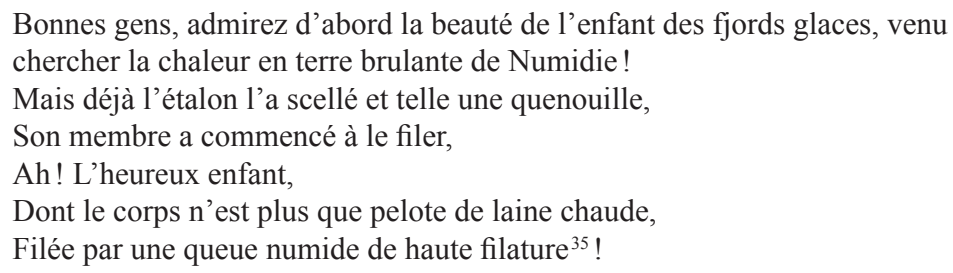

Peu de temps après, la voix du narrateur devient l'écho de Hawa : Ingvar sent « une queue lourde le filant, telle une quenouille, et son corps, ah! N'était plus alors qu'une pelote de laine chaude ${ }^{36}$. » De plus, Ingvar semble « interpellé personnellement » par un vers de Baudelaire : «Ô mort,

32. Mohamed Leftah, Hawa ..., op. cit., p. 17.

33. Ibid., p. 18-19.

34. Ibid., p. 121.

35. Ibid., p. 53.

36. Ibid., p. 57. 
vieux capitaine, il est temps! Levons l'ancre ", l'ancre en question étant bien entendu celle qui est tatouée sur le corps de Zapata.

Le corps produit par l'amour de Zapata et Ingvar, ainsi que l'amour incestueux de Zapata et de Hawa rappellent le Corps sans Organes (CsO) décrit par Deleuze, "le champ d'immanence du désir » qui se trouve dans le lieu où « le désir se définit comme processus de production, sans référence à aucune instance extérieure, manque qui viendrait le creuser, plaisir qui viendrait le combler ${ }^{37} \gg$. Parmi les possibilités développées par la réflexion deleuzienne se trouve celle de la nouvelle cartographie du corps : "Pourquoi pas marcher sur la tête, chanter avec les sinus, voir avec la peau, respirer avec le ventre ${ }^{38}$ ? » Le $\mathrm{CsO}$ conditionne le désir en stimulant de nouveaux assemblages créés par l'accouplement inouï d'organes et d'intensités, assemblages qui mettent en valeur le corps virtuel plutôt que le corps actuel. Ce qui est démontré par le chant des noces de Hawa et de Zapata, et les vœux de celui-ci :

\begin{abstract}
Oh, comme je vais baiser tes lèvres, et comme je vais baiser tes autres lèvres, toutes et sans exception.

Le lèvres de ton bas-ventre et celles, sombres et secrètes, si charnus, de ton dos, et les lèvres de tes seins, et les lèvres de tes yeux, ton corps ne sera plus que lèvres que je baiserai à tour de rôle, ensuite, passant d'une paire a l'autre, en même temps. Je m'enivrerai des parfums capiteux qu'exhalent toutes ces fleurs labiées qui parsèment ton corps!

Ô Hawa! Ton corps n'est plus que parterre fleuri et odorant de lèvres que je hume et baise sans me lasser ${ }^{39}$.
\end{abstract}

Le passage par le $\mathrm{CsO}$ déclenche un nouveau corps qui s'envole vers de nouveaux devenirs ${ }^{40}$.

Ce n'est pas par hasard que le décollage du corps a lieu à travers le chant. Leftah utilise le rapport entre la vie et l'art à des fins stratégiques. Car si le naturalisme est, selon Thomas Pavel, le style même de l'enracinement, selon lequel le destin de chaque personnage est déterminé par les lois, rigides et immuables, qui règlent son environnement, l'esthétisme est ce qui restitue à ce personnage son autonomie par rapport à ces forces sociales et économiques, le laissant dépendre d'un système de valeurs coupé de ce monde, à savoir les valeurs de l'art ${ }^{41}$. Si les personnages de Leftah

37. Gilles Deleuze et Félix Guattari, op. cit., p. 191.

38. Ibid., p. 187.

39. Mohamed Leftah, Hawa ..., op. cit., p. 41.

40. Les assemblages peuvent être plus cruels, comme le policier qui, en tenant une chaise par-dessus sa tête pour frapper les étudiants qui avaient occupé l'ambassade de France, acquiert quatre nouvelles mains. Mohamed Leftah, Une chute infinie, Paris, La Différence, 2009, p. 19-21, p. 27. Les devenirs qui résultent du passage par le CsO peuvent être eux-mêmes très violents, témoin le viol de Fatima qui « fait parler son lssan » mais qui est retourné contre son auteur, le commissaire Khabir, qui meurt égorgé.

41. Thomas Pavel, La Pensée du roman, Paris, Gallimard, 2003, p. 291-300, p. 359-371. 
chevauchent ces deux registres - le registre du corps et du demi-monde, et le registre de l'art et de l'esthétisme - c'est que Leftah tient à souligner l'importance des deux aspects suivants : la mise en valeur de la mondanité du corps et la corporalité de l'art. Les tatouages de Zapata et les chants de Hawa vont nécessairement de pair : le rapport incestueux liant le proxénète à sa sœur ne fait que raconter le pouvoir de l'art et sa place dans le monde. Nous ne sommes pas très loin de ce qu'Edward Said appelle « worldliness » ou mondanité : tout texte, toute œuvre d'art, existe dans le monde, à affaire aux forces de celui-ci, tout en étant simultanément un système autonome doté de sa propre poétique ${ }^{42}$. L'art sous toutes ses formes - peinture, chant, texte ou tatouage - existe comme lien social : on n'a pas affaire à l'autre sans la médiation de l'esthétique.

\section{Le corps dans l’État}

Si l'histoire de Zapata et de Hawa fonctionne comme une allégorie de l'esthétique de Leftah, une synthèse entre le corps et l'art, Le Jour de Vénus se déploie comme un essai sur les pouvoirs politiques du corps. Le commissaire Khabir, que nous avons vu dans la première partie de cet essai, tombe follement amoureux d'une féministe, Aïcha. De retour d'un congrès pendant lequel elle aurait tenu des propos qui inquiètent les autorités marocaines, elle est convoquée par les Renseignements généraux. En même temps, le beau-frère de Khabir, Jalal, qui est l'émir d'un groupuscule islamiste violent, décide d'enlever Aïcha : il trouve ses idées féministes trop dangereuses. Aïcha, son corps, sa beauté, ses idées, est celle par qui le scandale arrive.

Dès l'épigraphe de ce roman, tiré de Rimbaud, nous sommes dans une perspective qui revendique la matérialité et la supériorité du corps. Comme Rimbaud, Leftah est pris par l'idolâtrie de la chair :

Je crois en toi! Je crois en toi! Divine mère,

Aphrodite marine! Oh la route est amère,

Depuis que l'autre dieu nous attelle à sa croix

Chair, Marbre, Fleur, Vénus, c'est en toi que je crois!

Et c'est Aïcha qui jouera le rôle de cette déesse dans le roman.

La description de la cellule terroriste passe, elle aussi, par le corps. Leftah souligne l'importance de la métaphore corporelle dans le discours politique, mais c'est pour mieux révéler les dangers des «cellules » pareilles : « on peut méditer sur la signification à donner à ce recours du politique aux niveaux les plus élémentaires, les plus fondamentaux du vivant : la cellule, pour les partis communistes et d'une manière générale 
ceux qu'on pourrait appeler laïcs, la nawate, noyau, pour le mouvement islamiste $^{43}$. $\gg$ Ce que Leftah ne dit pas, mais que tout lecteur arabophone comprend, est que le noyau désigne la partie du fruit qui se crache et ne se mange pas. La cellule laïque est plus « vivante » que la nawate islamiste. Le chef de cette nawate a pour mission la diffusion dans le corps social de son propre message islamiste et soi-disant prophétique. Il s'agit donc d'introduire un corps étranger et pas forcément acceptable dans un corps politique. Étrangeté qui est doublée par le corps de Jalal lui-même :

\footnotetext{
L'apparence extérieure de Jalal était aux antipodes de celle qu'on imagine être celle d'un intégriste. À l'opposé de ces visages mangés par la barbe et au regard fiévreux, celui de Jalal était glabre, avec des joues roses et épanouies, des yeux d'un vert émeraude, chatoyants. Jalal n'utilisait ni souak, ni khôl, ni henné, ces cosmétiques féminins traditionnels, valorisés et sublimés par les " frères », mais des parfums subtils de firmes prestigieuses; de même pour l'habillement strict et élégant, griffé aux marques les plus célèbres. Jalal présentait l'apparence du cadre supérieur type du jeune loup ${ }^{44}$.
}

Leftah explique les choix politiques de Jalal par l'étrangeté de son corps. Il se trouve que Jalal descend d'une vieille famille andalouse, dont il a gardé les traits physiques. Sa mère, qui lui a transmis ces traits, lui a transmis aussi la légende familiale de son ascendance illustre, ainsi qu'un héritage lourd de reproches : Jalal est devenu cadre, il est riche, il a une famille mais il n'a pas de pouvoir politique. La seule solution qu'il peut proposer est de se proclamer émir, ne serait-ce que d'un groupuscule, pourvu qu'il garde le droit de commander à ses membres, et de recevoir l'allégeance de cinq sujets des classes les plus démunis ${ }^{45}$. Ainsi l'émirat de Jalal ne serait qu'une simple reproduction de n'importe quel système politique régressif du monde arabe.

Le jeune émir, affamé d'autorité et de pouvoir, entre en concurrence politique avec sa " victime », Aïcha. En montrant le portrait d'Aïcha à ses sujets, il dit : « Voici celle qui voudrait un jour vous gouverner », c'est-àdire celle qui pourrait un jour usurper la place qui revient de droit à Jalal, l'émir autoproclamé ${ }^{46}$. Il va de soi que l'idée qu'une femme puisse gouverner un État - que la tête du corps politique soit féminine - est totalement inacceptable. Jalal trouve catastrophique la prise de pouvoir par une femme, Bénazir Bhutto, au Pakistan : «Pour lui c'était une tache et une souillure sur le corps islamique qui ne pouvait être - corps biologique et corps politique que masculin, mâle, viril ${ }^{47}$. \ Il va de soi que quand la fatwa est lancée contre Aïcha, elle est articulée en termes corporels :

43. Mohamed Leftah, Le Jour de Vénus, Paris, La Différence, 2009, p. 59.

44. Ibid., p. 62.

45. Ibid., p. 64-65.

46. Ibid., p. 65.

47. Ibid., p. 57. 
Mes frères, je vous le dis en conscience, d'après notre chari'a, le sang de cette femme [...] vous est licite [...]. Son sang et son corps. Du moment qu'elle a renié l'islam, c'est une païenne qu'on peut capturer et traiter comme une Sabia (une captive que l'émir du jihad pouvait prendre pour concubine ou offrir à l'un de ses guerriers) ${ }^{48}$.

Le corps d'Aïcha deviendra ainsi le moyen de sa défaite et de son humiliation.

Car le corps d'Aïcha est un corps dangereux. Autant par sa beauté que par son pouvoir, Aïcha incarne la plus dangereuse des qualités, comme l'explique Leftah. Elle est fâtina, littéralement ensorcelante. Cet adjectif

appelle irrésistiblement un autre [...] fitna; dans l'acception coranique, guerrière (et guerre civile de surcroît) de ce mot : la fitna dont il est dit qu'elle est " pire que le meurtre » [cf. Le Coran, sourate II, v. 217]. Par cette connotation venue, issue du texte sacré, la fâtina, la femme belle, ensorcelante, loin d'apparaître aux yeux du rigoriste musulman comme une promesse du bonheur (la définition que donne Stendhal de la beauté) est appréhendée par lui au contraire comme un être gros de menaces, de subversion pour la stabilité et l'harmonie de l'ordre social ${ }^{49}$.

Cette déstabilisation de l'ordre social commence tôt : le commissaire Khabir, converti aux idées intégristes de son beau-frère, oscille entre des déclarations d'admiration et celles d'un ordre plus vulgaire (« Putain! Sale pute! ») qu'il hurle en lisant le dossier d'Aïcha aux Renseignements généraux. Une simple photographie d'Aïcha déclenche une perte de contrôle totale chez le commissaire. Il en va de même pour les trois membres du groupe de Jalal, qui sont pris entre l'enchantement de la fâtina et les passions plus dangereuses produites par la fitna, les deux mots qui « fusionnaient et se matérialisaient dans le visage et le corps d'une femme : Aïcha ${ }^{50} »$.

La rencontre entre Aïcha et Jalal après l'enlèvement ne fait que reconfirmer ce système de valeurs produit par le couple fitnalfâtina, tout en le retournant contre Jalal. L'émir explique à sa victime qu'il ne souscrit à aucune définition occidentale de la beauté. "La beauté, quand elle s'épanouit sauvagement et sans entraves, hors du cadre de la chari'a, est fitna, subversion et corruption, et doit être combattue en conséquence ${ }^{51}$. » La réponse d'Aïcha révèle la faiblesse de l'argument de Jalal : «Dans ce cas toi aussi tu dois être combattu et châtié, puisqu'il faut le reconnaître ta beauté est une véritable fitna ${ }^{52}$. » La réponse d'Aïcha ne tient pas compte, malheureusement, de l'extrême misogynie de Jalal.

\footnotetext{
48. Ibid., p. 66.

49. Ibid., p. 71-72.

50. Ibid.

51. Ibid., p. 88.

52. Ibid., p. 88.
} 
Après ce moment les événements passent vite : Aïcha est mutilée, ses cheveux sont coupés, mais elle est sauvée par le commissaire, qui, à force de lire son dossier et de regarder sa photo, tombe amoureux de la fâtina. À l'hôpital elle se perd dans une dépression nerveuse. Son amant, le psychanalyste Safwan, se lie d'une amitié presque fraternelle avec le commissaire. Le roman se termine par une citation de Baudelaire :

\footnotetext{
Que tu viennes du ciel ou de l'enfer, qu'importe

Ô Beauté! Monstre énorme, effrayant, ingénu!

Si ton œil, ton souris, ton pied, m'ouvrent la porte

D'un infini que j'aime et n'ai jamais connu.
}

La beauté du corps d'Aïcha, loin de faire scandale, accomplit l'impossible, ou plutôt ce qu'on n'a jamais connu : elle crée paix et unité là où il n'y avait que haine et division dans le corps social.

\section{Conclusions}

L'enquête littéraire sur les pouvoirs du corps constitué par les écrits de Leftah nous mène loin de la doxa de ce sujet. À travers les corps tatoués, mutilés, torturés et violés, Leftah pousse son lecteur vers une considération de la signification et de la création littéraire, pour lui rappeler que l'une ne va jamais sans l'autre, que la signification est forcément corporelle et matérielle avant d'être spirituelle. Ce rappel de la matérialité du corps permet une critique radicale des institutions de l'État - familles, écoles, autorités politiques et religieuses - qui vise à mettre en valeur la fragilité de l'individu face à la violence des institutions qui le déterminent. Ce n'est que dans l'ordre poétique - un terme qui doit comprendre tous les genres littéraires, et qui est le seul que Leftah trouve acceptable - que l'individu peut jouir d'une pleine possession de ses droits, de sa langue, de son corps.

Ziad Elmarsafy Department of English and Related Literature, University of York 